\title{
PERSPECTIVE OPEN Sustainable sanitation and gaps in global climate policy and financing
}

\author{
Sarah Dickin $\mathbb{D}^{1 凶}$, Moustafa Bayoumi ${ }^{2}$, Ricard Giné $\mathbb{D}^{3}{ }^{3}$, Kim Andersson ${ }^{1}$ and Alejandro Jiménez (iD ${ }^{3}$
}

Although sanitation systems are fundamental for human health and sustainable development, limited focus has been placed on their contributions to climate mitigation and adaptation. Climate change threatens existing systems, as well as efforts to increase services for 2.3 billion people who lack even a basic sanitation service. At the same time, the sanitation and wastewater sector directly produces emissions associated with breakdown of organic matter, and treatment processes require large energy inputs. In light of these challenges, we describe gaps in how sanitation is being addressed in mitigation and adaptation, discuss how this results in little inclusion of sanitation in climate policy and financing at the global level, and implications of these gaps for different sanitation systems and geographic regions. Finally, we describe the need for planning frameworks to facilitate integration of climate change into sanitation policy and programming. This will be critical to increasing understanding of sanitation and climate change linkages among stakeholders, and more effectively including sanitation in climate action.

npj Clean Water (2020)3:24; https://doi.org/10.1038/s41545-020-0072-8

\section{INTRODUCTION}

The Sustainable Development Goal (SDG) target 6.2 is achievement of universal access to a 'safely managed' sanitation facility and elimination of open defecation. The effort required to achieve this target is significant, as $\sim 5.6$ billion more people will need to use safely managed services, and $\sim 1.3$ billion will need to shift from open defecation to the use of a sanitation system by $2030^{1}$. According to the Joint Monitoring Programme, 'safely managed' services refers to 'use of an improved type of sanitation facility that is not shared with other households and the excreta produced must either be safely treated in situ, or transported and treated off-site ${ }^{2}$. Closely linked is SDG target 6.3, which addresses the need for greater wastewater treatment, as the majority of wastewater is currently discharged untreated ${ }^{3}$.

Climate change impacts existing sanitation systems and impedes progress to achieving these targets by increasing variability in the water cycle, operating through increasingly intense climate events, unpredictability of water availability and decreasing water quality ${ }^{4,5}$. These impacts also exacerbate existing challenges in the sector, such as sustainability concerns related to infrastructure breakdowns. Heavy precipitation and flood events can lead to physical damage of sanitation infrastructure, as well as indirect damages from flooding, such as impacts to transportation and energy supply that may disrupt regular operation and maintenance of systems ${ }^{6}$. In coastal areas, sea-level rise is also predicted to increase flooding ${ }^{7}$. Pit latrines and septic systems become non-functional when filled with water ${ }^{8}$. They may collapse or experience damage to the above-ground structures during flood or storm events making them unusable, with users forced to practice open defecation ${ }^{6}$. These events also impact collection and transportation systems for faecal sludge, particularly in densely populated urban areas and informal settlements.

Reduced precipitation and limited water availability impacts piped sewer systems, as these systems rely on adequate water to remove waste. Limited water availability due to drought can increase pollutant concentration resulting in more contaminated wastewater, which has greater impacts when discharged untreated into the environment. In addition, high temperatures, heavy rainfall, flooding and drought events can modify the distribution of diarrhoeal diseases ${ }^{9}$. These sanitation-related health effects contribute to undermining gains made in public health over the last several decades ${ }^{10}$.

These wide-ranging impacts require careful consideration of adaptation options within the sector that are not limited to technical considerations. Increasing access to sanitation and wastewater treatment is also critical to consider from the perspective of climate mitigation where emissions are growing and have been overlooked. Despite the importance of climate change to the sanitation sector, there are gaps in how both mitigation and adaptation are being addressed. We describe these limitations, and ways that these gaps have resulted in little inclusion of sanitation climate policy, programming and financing.

\section{Closing the sanitation gap while considering contributions to} mitigation action

Sanitation and wastewater systems contribute to greenhouse-gas emissions (GHG) both directly through breakdown of excreta discharged into the environment or during treatment processes, and indirectly through the energy required for treatment steps. Most attention to emissions from sanitation and wastewater systems has been on centralised treatment plants, which require considerable energy input and are estimated to account for $3 \%$ of global electricity consumption. In addition, degradation of organic matter during wastewater treatment contributes $\sim 1.57 \%$ of global GHG emissions and 5\% of global non-carbon dioxide greenhousegas emissions ${ }^{11,12}$. Although there is evidence that GHG emissions from biological processes in wastewater treatment plants are major GHG contributors in some countries, they remain poorly understood due to lack of the data availability and model complexity ${ }^{13}$. This information will be increasingly critical as countries move towards increasing coverage of wastewater treatment. For instance, in China, urbanisation and associated

\footnotetext{
'Stockholm Environment Institute, Linnégatan 87D, 10451 Stockholm, Sweden. ${ }^{2}$ Department of Earth Sciences, Uppsala University, Villavägen 16, SE-752 36 Uppsala, Sweden.




growth in the number of wastewater treatment plants has led to an increase in domestic wastewater emissions of $400 \%$ from 2000 to $2014^{14}$. In the case of GHG emissions modelled for the Metropolitan area of Santiago, Chile, these are projected to reach around $50 \%$ of national emissions by 2027 with increasing production and treatment of wastewater and sludge ${ }^{15}$. In addition to treatment processes, there is potential to reduce energy use in sewerage conveyance, such as through gravity-based systems and increased use of distributed or decentralised systems that reduce pumping distances ${ }^{16}$. As countries expand their wastewater systems and treatment capacities or replace ageing facilities, decisions made today will have long-lasting consequences for climate action.

Discharge of untreated waste into the environment and the use of on-site technologies, such as septic systems and pit latrines, are also sources of emissions but remain poorly quantified ${ }^{17,18}$, despite the expected increases in access to these technologies promoted by SDG target 6.2 . For example, $\sim 80 \%$ of the global emissions of $\mathrm{N}_{2} \mathrm{O}$ from human waste are from uncollected waste, a common occurrence in regions where the majority of the population is not connected to a sewage system with wastewater treatment ${ }^{19}$. The widespread use of pit latrines provides one example showing the importance of evaluating the GHG impacts of decentralised sanitation technologies. Pit latrines are one of the main sanitation options for low-income countries as they are hygienic, low-cost and easy to construct, and around 1.77 billion people rely on them on a daily basis ${ }^{20}$. Pit latrines are estimated to account for $\sim 1 \%$ of global anthropogenic methane emissions ${ }^{17}$. Although relatively small compared with other GHG sources, these emissions require consideration in planning of sanitation and wastewater systems. Providing sanitation access to 800 million people in India through pit latrines would cause increases in India's annual GHG emissions, equivalent to $7 \%$ of current levels ${ }^{21}$. The climate impact of providing 2.3 billion people who still lack access to basic sanitation with services such as pit latrines could more than double the GHG emissions from this source ${ }^{22}$. Pit latrines and similar on-site anaerobic technologies that produce methane are poorer choices from an emissions perspective than aerobic technologies, such as properly operated composting toilets $^{17,23}$. Additional measures to reduce GHG emissions resulting from the breakdown of excreta include regular emptying of septic tanks ${ }^{24}$ and good wastewater management ${ }^{16}$. Despite these concerns, emissions are rarely considered by countries or development organisations in the selection of technologies to increase access and close the sanitation gap. In the context of rapid urbanisation and the need to scale up sanitation, research is needed to facilitate comparison of absolute and relative emissions of the available sanitation options to assist in future decisions on technologies ${ }^{25}$.

In making progress towards SDG targets 6.2 and 6.3 , there is significant potential to reduce emissions from sanitation and wastewater systems through recovery of energy and nutrients contained in waste ${ }^{11}$, while also providing indirect reduction of emissions through renewable energy production and reduced dependency on fossil-based chemical fertilisers ${ }^{26}$. This would contribute not only to climate action and reduced discharge of contaminants into the environment but also provide co-benefits through food and energy security. For instance, on a global scale, the phosphorus available from human excreta, if collected, could equal $22 \%$ of total global phosphorus demand ${ }^{27}$. Substituting $5 \%$ of the existing nitrogen fertiliser production with nitrogen recovered from human waste would save more than $1.5 \%$ of China's annual electricity consumption ${ }^{28}$. In addition, energy recovery from wastewater systems could provide over $55 \%$ of the electricity required for municipal wastewater treatment by $2040^{29}$, demonstrating the dual benefits of reducing waste-related emissions and producing renewable energy.
Broadening understanding of adaptation action in the sanitation sector

Although climate impacts on sanitation systems present global risks, low-income countries face greater risks in many cases, however, attention to adaptation needs within the sanitation sector has been limited ${ }^{30}$. To date, adaptation strategies have focused on 'additional' technical components of sanitation infrastructure related to particular risks that can be attributed to climate change ${ }^{31}$. Pit latrines can be constructed in a raised manner, using special coatings, or with smaller or shallower size of pits to improve ability to withstand flood events and reduce contamination in the case of collapse $e^{32}$. Ensuring separation of stormwater from wastewater is also promoted to reduce risks related to overflows or damage to collection and treatment infrastructure ${ }^{33}$. In water scarce regions, water saving and reuseoriented sanitation technologies with lower dependence on water supply are expected to have greater resilience . $^{8}$

The focus on specific 'hard' adaptation measures to upgrade existing sanitation technologies has been reinforced by eligibility for climate finance, which has often been limited to these additional infrastructure elements. Such 'hard' components are more easily measurable as 'additional', while 'soft' measures are not so visible ${ }^{34}$. As a result, technological solutions have been prioritised over 'soft' adaptation measures, such as improved planning, institutional and regulatory arrangements, capacity building, monitoring, public awareness or behavioural responses ${ }^{31}$. Rather than focus on additionality that privileges certain hard interventions, a greater focus should be placed on identifying the most efficient, effective and equitable adaptation measures within the sanitation sector, thus identifying measures with the lowest possible costs, most effective in reducing negative climate impacts, and that reach the most vulnerable populations and countries ${ }^{35}$.

This requires greater consideration of soft adaptation strategies that go beyond technocratic responses ${ }^{36}$. For instance, strengthening capacity of wastewater system managers to address climate change risks is needed to overcome knowledge gaps that may limit adaptation ${ }^{37}$. Developing effective information systems can play a significant role in ensuring that sanitation workers and users can access updated and reliable data, which are needed to make informed decisions to ensure services are maintained. However, sanitation is often missed or excluded from detailed consideration in national assessments of climate change impacts, which hampers regular data collection and reporting ${ }^{36}$.

The large gap in sanitation access demonstrates the importance of better integration of adaptation with development interventions. Poverty and underdevelopment are strongly interlinked with vulnerability to climate change ${ }^{38}$, and essential services are a priority for reducing vulnerability ${ }^{39}$. The IPCC Fifth Assessment Report (AR5) suggests that the most effective actions to reduce vulnerability in the short-term relate to the implementation of 'basic public health measures such as provision of clean water and sanitation... and alleviate poverty (very high confidence) ${ }^{139}$. This indicates a much broader role for essential services in climate adaptation, particularly among the most vulnerable, indicating that investments in basic sanitation infrastructure or behaviour change focused sanitation initiatives need to be promoted to shift 1 billion people from practising open defecation to more resilient services. Such interventions, however, are not commonly viewed as adaptation. This division between adaptation and development has limited mainstreaming of climate considerations into sanitation planning, which may be locking countries into vulnerable development paths.

The concept of adaptive development that promotes a greater focus on risk management in development is highly relevant for sanitation interventions ${ }^{38}$. For instance, sanitation plays a fundamental role in adapting to altered incidence of waterborne 
diseases, particularly diarrhoeal diseases ${ }^{9}$. Risks are greatest for those with the lowest levels of sanitation services. This is particularly critical during extreme events, although challenges in climate attribution may have limited stakeholder awareness of these risks ${ }^{40,41}$. Sanitation interventions can also address structural inequalities contributing to inequal distribution of climate change vulnerability, as there is increasing focus in the sector on targeting countries and population groups in greatest need of assistance ${ }^{42}$. As inadequate sanitation disproportionately impacts women, poor and marginalised communities, and remote regions such as the Arctic $^{43}$, sanitation-related climate adaptation offers synergies in addressing inequalities. By building management of climate risks into sanitation development plans, adaptation action can make greater contributions to poverty alleviation and reduction of inequalities ${ }^{34}$. As funds for addressing climate change are currently, and will continue to be, substantial in comparison to funds for sanitation investments, it is critical to consider sanitation within broader climate adaptation.

Sanitation missing from the climate policy debate

The range of mitigation and adaptation opportunities related to sanitation and wastewater systems indicate that opportunities for climate action are overlooked, as there is very limited inclusion in climate policy and finance. To understand where sanitation is situated in the climate policy debate, it is helpful to examine how it is included in the nationally determined contributions (NDCs). Although the NDCs are non-binding, they provide an indication of national priorities and interests of a contributing country related to mitigation and adaptation. Water is one of the top five sectors described as vulnerable to climate change within the NDCs, as it is identified by 100 countries $^{44}$. Despite this focus, limited concrete action has been proposed related to sanitation ${ }^{45}$. The SDG-NDC connections tool analyses and quantifies the points of connection related to mitigation or adaptation activities included in the NDCs and the SDGs (at the level of goals and individual targets), including those related to access to safe sanitation and wastewater treatment ${ }^{46}$. Our analysis of the SDG-NDC connections tool data, focusing on SDG 6-related NDCs, showed that only $2 \%$ of these NDCs deal with sanitation access, while wastewater management is mentioned in $3 \%$ (Fig. 1).

a

SDG 6-related activities in NDCs



- All other SDGs $\backsim$ SDG6 activities
A further examination of the activities related to sanitation access and wastewater management shows a majority of the activities are adaptation activities, with few mitigation activities. Sanitation and domestic wastewater activities in the NDCs are reported largely by lower-middle-income countries, with very limited inclusion by high-income countries (Fig. 2a). Geographical trends can also be observed (Fig. 2b), as the Middle East and North Africa (MENA) region and Sub-Saharan Africa had the highest number of activities related to sanitation and wastewater. This may be due to extensive experience of water reuse and treatment in the MENA region, and the current water scarcity challenges facing both regions.

It is also notable that no sanitation-related mitigation activities are included by USA, Indonesia, India or China, despite these countries accounting for almost half of global recorded methane emissions from wastewater, and half of recorded nitrous oxide emissions from domestic wastewater in $2000^{47}$. A limited inclusion of sanitation activities within the NDCs suggests that national decision-makers may not see a role for sanitation within climate action or have limited evidence of these linkages.

Is climate finance part of the problem or the solution?

The capital investments needed to meet the SDGs targets 6.1 and 6.2 for safe water and sanitation are approximately three times the current investment levels ${ }^{48}$. These financing challenges highlight where current spending is insufficient to meet the SDG targets by 2030, but at the same time, it is important to consider how these investments will be made in ways that contribute to climate action. Climate finance offers an opportunity to address these investment needs through sanitation focused climate action; however, this has been very limited so far.

An analysis conducted by the authors of Approved Project Proposals by the Green Climate Fund (GCF) board up until April 1, 2019 indicates a limited number of projects focused on or related to sanitation and wastewater. Out of 99 projects approved so far by the GCF board, only 7 had a specific sanitation or wastewater element. Furthermore, these sanitation elements were mostly funded by development banks or national governments rather than the GCF itself. Excluding two projects that had no clear financial amount allocated for the sanitation component, the b Sanitation and wastewater in SDG 6-related activities



- All other themes - Access to sanitation - Wastewater treatment

Fig. 1 NDC activities related to SDG 6. a The SDG-NDC connections tool indicates 630 out of 6900 activities (9\%) were identified related to SDG 6. b Within the SDG 6-related activities, $2 \%$ were linked with access to sanitation and $3 \%$ to wastewater treatment ${ }^{46}$. Other activities in SDG 6 relate to water management, water access and supply, improved irrigation. 


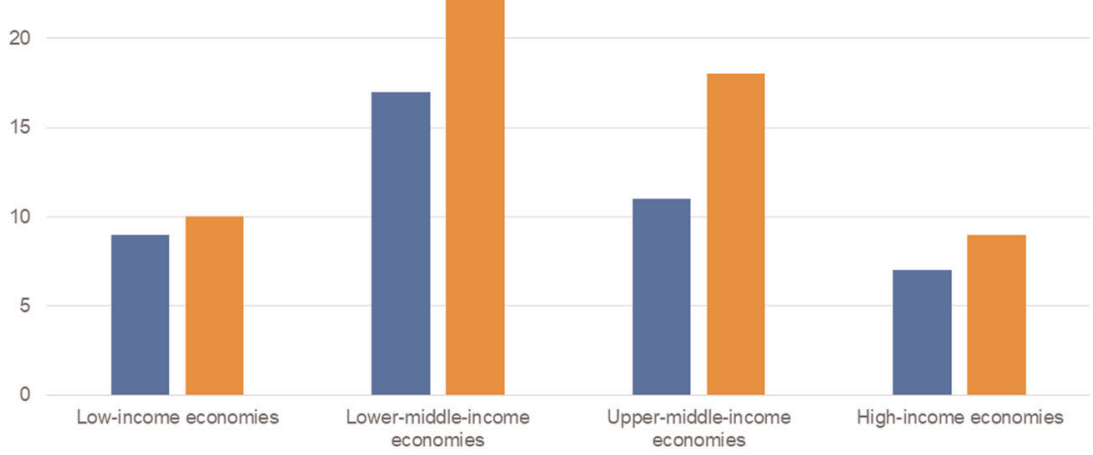

b - Countries $=$ Activities

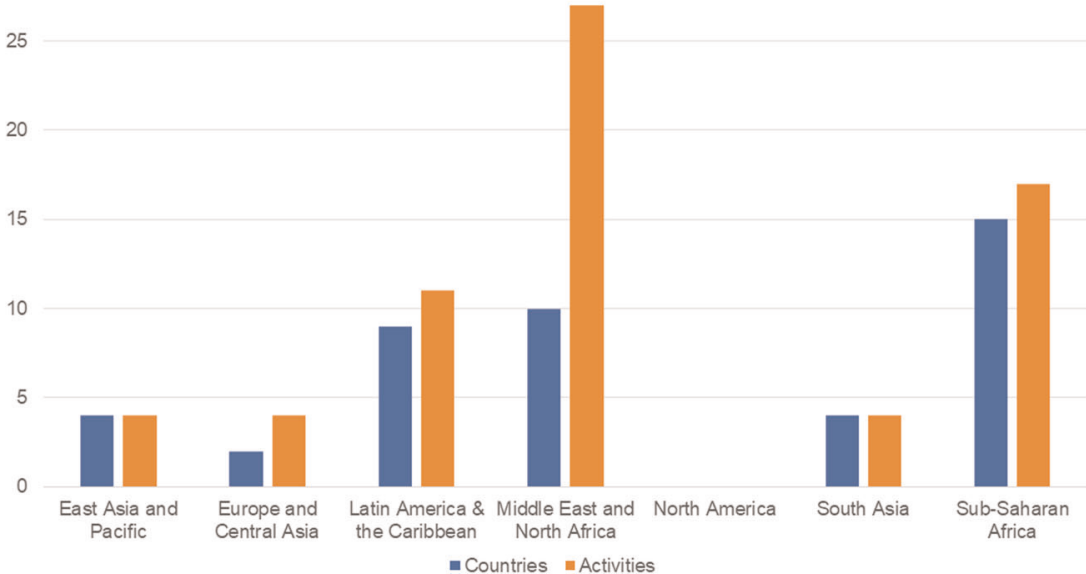

Fig. 2 Sanitation and wastewater activities included in the NDCs. a Displayed by World Bank country income classifications. b Displayed by region.

other five projects received USD 1.24 million from the GCF out of a USD 5 billion budget allocated to approved projects. This accounts for $<0.025 \%$ of the GCF approved project budgets.

Examining climate-related Official Development Assistance (ODA) data from the OECD shows a similar trend. In 2017, projects related to water supply and sanitation with climate change as a main objective saw commitments of USD 938 million $^{49}$. In the same year, projects targeting mitigation and adaptation related to basic sanitation and large sanitation systems received USD 29 million. This represents 3\% of climate-related finance for the water supply and sanitation sector. While there are other codes such as 'Basic water supply and sanitation' that also include sanitation projects, research has shown that there is a dilution of sanitation within drinking-water and sanitation ODA finance ${ }^{50}$. This database is thought to reflect large overestimations, with far fewer projects than what is reported likely to be relevant to climate change mitigation and adaptation ${ }^{51}$.

Limited examples of climate finance related to sanitation indicate an overlooked source of funds for reaching SDG 6.2 and 6.3. Aside from the investments needed, there are large economic losses associated with unsafe water supply and sanitation, which are estimated to be $1.5 \%$ of global Gross Domestic Product (GDP) ${ }^{52}$. This number is even higher in certain regions where it exceeded 2\% of total GDP in East Asian and Pacific and Sub-Saharan African economies, while in South Asia, it exceeded $4 \%$ of GDP ${ }^{52}$. With substantial economic co-benefits and an urgency to adapt to climate change while reducing emissions, there is a need to promote greater opportunities for climate finance to complement development finance in the sanitation sector.

This is especially important in least developed countries with high vulnerability to climate change impacts and limited adaptive capacity, while facing major sanitation challenges such as widespread open defecation. Such countries typically have a very small national budget to invest in sanitation. For example, Liberia has an open defecation rate of $62 \%$ in rural areas, and $20 \%$ in urban areas $^{42}$, while wastewater treatment is not available in the country. The total average spending for WASH sector in the last 
3 years is around $37 \mathrm{M}$ USD/year, with government spending below $2 \mathrm{M}$ USD/year ${ }^{53}$. In comparison, a small-scale GCF project budget is above USD 10 million and up to USD 50 million, and a proposal to the GCF costs around USD 1 million to prepare ${ }^{54}$. Such examples indicate that climate finance could make a major contribution to adaptive development in the sanitation sector; however, two obstacles are the investment and capacity needed to formulate projects, and the narrow focus on 'additional' sanitation components as adaptation measures.

\section{Discussion and future directions}

Sanitation has long been acknowledged as fundamental for health and well-being; however, its contributions to other aspects of sustainable development are more recently being recognised. The emphasis on integration throughout the SDG Agenda has highlighted that targets for sanitation and increased wastewater treatment must be achieved in order to attain a number of other outcomes, including good water quality, healthy aquatic ecosystems, gender equality and health and well-being ${ }^{55}$. At the same time, contributions to climate action have been overlooked, limiting policy coherence with the SDG agenda and other global agreements that address systemic risks, particularly the Paris Agreement and the Sendai Framework on Disaster Risk Reduction. The Sendai Framework Target 4 focuses on 'Substantially reduce disaster damage to critical infrastructure and disruption of basic services,' which requires attention to sanitation systems. The limited inclusion of sanitation within the NDCs and climate finance indicates little awareness of the linkages between sanitation and climate change among stakeholders, and a need to strengthen this evidence in terms of impacts, as well as mitigation and adaptation opportunities. Assessment of climate change impacts on sanitation systems has focused on technical challenges, with a need for broader information on sanitation-related climate change impacts on social systems. This includes a more detailed understanding of the links between sanitation, health and climate change, including modifications of waterborne and vector-borne disease prevalence, increases in antimicrobial resistance and mental health and well-being implications, such as increased stress, potential exposure to violence and anxiety from lack of access to toilet facilities ${ }^{36,56}$. A more comprehensive evidencebase will support planning of locally appropriate sanitation and wastewater systems that take into account a broader range of climate impacts. For development organisations, continued focus on 'business as usual' to meet immediate needs risks locking users into unsustainable systems in the face of future uncertainties ${ }^{16}$. Current choices for sanitation technologies will be strong determinants of sector emissions and climate resilience in the future ${ }^{33}$.

More systematic assessment of GHG emissions from different sanitation technologies is needed to better inform decisionmaking in the sector ${ }^{13,23}$, whether it involves selection of on-site technologies in low-income countries or upgrading of wastewater treatment plants in high-income countries. Moving from coarse estimates, such assessments must account for the role of contextspecific factors, such as high water tables that increase the anaerobic zones of pit latrines, or the carbon intensity of an electricity grid that may drive interest in energy recovery from wastewater treatment ${ }^{17,57}$. This evidence will inform the development and improvement of tools to support transition towards a more climate neutral water and sanitation sector. One such tool is the 'Energy Performance and Carbon Emissions Assessment and Monitoring' (ECAM) Tool developed by the Water and Wastewater Companies for Climate Mitigation (WaCCliM) project. The ECAM tool can assess carbon emissions for the urban water cycle, within the boundaries of utilities, in order to demonstrate how the sector can reduce greenhouse-gas emissions, both in the short-term (e.g., improving operational efficiency) and the long-term (e.g., reforming water infrastructure $)^{58}$. Similar tools could facilitate low-carbon sanitation development beyond the jurisdiction of water and wastewater utilities, e.g., for pit latrines and other on-site solutions.

A more comprehensive understanding of adaptation within the sanitation sector is needed, due to close links to poverty and vulnerability, which inextricably link it to development ${ }^{25}$. Sanitation is a prime example of the need for a broader view of adaptation that considers the complexity of ensuring equitable access to essential services for the poorest communities while adapting to a range of climate change risks. Greater awareness of this connection is needed among stakeholders to facilitate more opportunities for sanitation-related climate finance, and particularly projects that ensure equitable access to sanitation through resilient and low emission systems. This includes better opportunities for adaptation in the short-term targeted to 'soft' activities, particularly easy-to-implement changes in practices, that reduce vulnerability and increase adaptive capacity ${ }^{34}$. It is unclear whether the limited number of approved sanitation projects in the GCF portfolio is because climate finance organisations have been less likely to fund sanitation components, or whether countries have limited ability to apply for sanitation-related climate finance due to a lack of adequate capacity and contextspecific evidence, but strategies to build greater awareness from both sides are needed. Considering the shortfall in current investment needed to achieve SDG 6.2, especially in least developed countries, climate finance can play an important role in narrowing the sanitation access gap and improving the resilience of current systems.

Moving forward guidance is needed to support decision-makers in systematically and effectively addressing the challenges increasingly presented by climate variability and change, identifying areas in need of further support and articulating costed and prioritised plans. Applying principles drawn from broader climate change literature has been proposed to inform sanitation planning, such as adaptive management, consideration of complex system dynamics and flexibility and diversified risk ${ }^{31}$. A framework that provides key principles for addressing WASH and climate change is the 'Strategic Framework for WASH Climate Resilience' developed by GWP and UNICEF. This framework seeks to advance sector thinking around WASH and climate change, focusing on the planning and execution of actions to promote climate resilience in WASH strategies, plans and approaches ${ }^{59}$. A further opportunity is expanding the WHO Sanitation Safety Planning approach (SSP). SSP provides a structure to bring together actors to conduct local level assessment and management of health risks across the sanitation chain, which could be expanded to include climate considerations more comprehensively, beyond the identification of hazardous events related to seasonal or climatic factors. Similarly, adapting existing frameworks to address sanitation is an opportunity to draw on lessons from other sectors, such as the operational framework developed by $\mathrm{WHO}$ for building climate-resilient health systems ${ }^{60}$. This approach focuses on identifying key health systems functions where climate resilience can be strengthened, in order to anticipate, prevent and manage climate-related health risks. These and other frameworks provide a good starting point, but more research is needed to adapt them specifically to a sanitation and climate focus, and to operationalise and evaluate the frameworks across different development contexts. In addition, planning frameworks require not only a focus on climate-resilient technologies, but addressing the limitations in mitigation and adaptation highlighted here, to ensure that sanitation effectively contributes to climate action. 


\section{DATA AVAILABILITY}

The NDC-SDG connections data that support the findings of this study are available from https://klimalog.die-gdi.de/ndc-sdg/, which were obtained for this study and so are not publicly available. The data are, however, available from the authors upon reasonable request and with permission of SDG-NDC connections. The GCF data sets generated and analysed during this study are available from the corresponding author on reasonable request.

Received: 14 November 2019; Accepted: 16 April 2020;

Published online: 25 May 2020

\section{REFERENCES}

1. Mara, D. \& Evans, B. The sanitation and hygiene targets of the sustainable development goals: scope and challenges. J. Water, Sanit. Hyg. Dev. 8, 1-16 (2017).

2. WHO/UNICEF JMP. Progress on Drinking Water, Sanitation and Hygiene: 2017 Update and SDG Baselines (WHO and UNICEF, Geneva, 2017).

3. WHO and UN-HABITAT. United Nations. Progress on Safe Treatment and Use of Wastewater: Piloting the monitoring methodology and initial findings for SDG indicator 6.3.1 (WHO and UN-HABITAT, Geneva, 2018).

4. UN-Water. Climate Change and Water (UN Water, Geneva, 2019).

5. Jiménez Cisneros, B. E. et al. Freshwater resources. In Climate Change 2014: Impacts, Adaptation, and Vulnerability. Part A: Global and Sectoral Aspects. Contribution of Working Group II to the Fifth Assessment Report of the Intergovernmental Panel on Climate Change (eds Field, C.B. et al.), pp 229-269 (Cambridge University Press, Cambridge, UK and New York, NY, USA, 2014).

6. Sherpa, A. M., Koottatep, T., Zurbrügg, C. \& Cissé, G. Vulnerability and adaptability of sanitation systems to climate change. J. Water Clim. Chang 5, 487-495 (2014).

7. Hadwen, W. L. et al. Putting WASH in the water cycle: climate change, water resources and the future of water, sanitation and hygiene challenges in Pacific Island countries. J. Water Sanit. Hyg. Dev. 5, 183-191 (2015).

8. Luh, J., Royster, S., Sebastian, D., Ojomo, E. \& Bartram, J. Expert assessment of the resilience of drinking water and sanitation systems to climate-related hazards. Sci. Total Environ. 592, 334-344 (2017).

9. Levy, K., Smith, S. M. \& Carlton, E. J. Climate change impacts on waterborne diseases: moving toward designing interventions. Curr. Environ. Heal. Rep. 5, 272-282 (2018).

10. Cole, J. Sanitation in the Context of Planetary Health: Opportunities and Challenges (Secretariat of the Rockefeller Foundation Economic Council on Planetary Health, Oxford, 2018).

11. Li, W.-W., Yu, H.-Q. \& Rittmann, B. E. Chemistry: reuse water pollutants. Nature 528, 29-31 (2015).

12. Lu, L., Guest, J. S. \& Peters, C. A. et al. Wastewater treatment for carbon capture and utilization. Nat. Sustain 1, 750-758 (2018).

13. Mannina, G. et al. Greenhouse gases from wastewater treatment-a review of modelling tools. Sci. Total Environ. 551-552, 254-270 (2016).

14. Du, M. et al. Estimates and predictions of methane emissions from wastewater in China from 2000 to 2020. Earth's Futur. 6, 252-263 (2018).

15. Préndez, M. \& Lara-González, S. Application of strategies for sanitation management in wastewater treatment plants in order to control/reduce greenhouse gas emissions. J. Environ. Manag. 88, 658-664 (2008).

16. Carrard, N. \& Willetts, J. Environmentally sustainable WASH? Current discourse, planetary boundaries and future directions. J. Water Sanit. Hyg. Dev. 7, 209-228 (2017).

17. Reid, M. C., Guan, K., Wagner, F. \& Mauzerall, D. L. Global methane emissions from pit latrines. Environ. Sci. Technol. 48, 8727-8734 (2014).

18. Bogner, J. et al. Waste Management, in Climate Change 2007: Mitigation. Contribution of Working Group III to the Fourth Assessment Report of the Intergovernmental Panel on Climate Change (Cambridge University Press, Cambridge, 2007).

19. Strokal, M. \& Kroeze, C. Nitrous oxide $\left(\mathrm{N}_{2} \mathrm{O}\right)$ emissions from human waste in 1970-2050. Curr. Opin. Environ. Sustain 9-10, 108-121 (2014).

20. Graham, J. P. \& Polizzotto, M. L. Pit latrines and their impacts on groundwater quality: a systematic review. Environ. Health Perspect. 121, 521-530 (2013).

21. Kulak, M., Shah, N., Sawant, N., Unger, N. \& King, H. Technology choices in scaling up sanitation can significantly affect greenhouse gas emissions and the fertiliser gap in India. J. Water Sanit. Hyg. Dev. 7, 466-476 (2017).

22. van Eekert, M. H. A. et al. Anaerobic digestion is the dominant pathway for pit latrine decomposition and is limited by intrinsic factors. Water Sci. Technol. 79, 2242-2250 (2019).

23. Orner, K. D. \& Mihelcic, J. R. A review of sanitation technologies to achieve multiple sustainable development goals that promote resource recovery. Environ. Sci. Water Res. Technol. 4, 16-32 (2018).

24. Eggelston, H. S., Buendia, L., Miwa, K., Ngara, T. \& Tanabe, K. (eds). In 2006 Guidelines for National Greenhouse Gas Inventories, Wastewater Treatment and
Discharge (IPCC National Greenhouse Gas Inventories Programme, Hayama, 2006).

25. Howard, G., Calow, R., Macdonald, A. \& Bartram, J. Climate change and water and sanitation: likely impacts and emerging trends for action. Annu. Rev. Environ. Resour. 41, 253-276 (2016).

26. Sigurnjak, I. et al. Utilization of derivatives from nutrient recovery processes as alternatives for fossil-based mineral fertilizers in commercial greenhouse production of Lactuca sativa L. Sci. Hortic. 198, 267-276 (2016).

27. Mihelcic, J. R., Fry, L. M. \& Shaw, R. Global potential of phosphorus recovery from human urine and feces. Chemosphere 84, 832-839 (2011).

28. Wan, J., Gu, J., Zhao, Q. \& Liu, Y. COD capture: a feasible option towards energy self-sufficient domestic wastewater treatment. Sci. Rep. 6, 25054 (2016).

29. Water, I. E. A. Energy Nexus: Excerpt from the World Energy Outlook 2016 (IEA, Paris, 2017).

30. Oates, N., Ross, I., Calow, R., Carter, R. \& Doczi, J. Adaptation to Climate Change in Water, Sanitation and Hygiene: Assessing Risks and Appraising Options in Africa (ODI, London, 2014).

31. Mills, F., Kohlitz, J., Carrard, N. \& Willetts, J. Considering Climate Change in Urban Sanitation: Conceptual Approaches and Practical Implications [USHHD Learning Brief] (SNV, The Hague, 2019).

32. Morshed, G. \& Sobhan, A. The search for appropriate latrine solutions for floodprone areas of Bangladesh. Waterlines 29, 236-245 (2010).

33. WHO. Guidelines on Sanitation and Health (WHO, Geneva, 2018).

34. Fankhauser, S. \& Burton, I. Spending adaptation money wisely. Clim. Policy 11, 1037-1049 (2011).

35. Stern, N. The economics of climate change. Am. Econ. Rev. 98, 1-37 (2008).

36. WHO. Climate, Sanitation and Health (WHO, Geneva, 2019).

37. Kirchhoff, C. J. \& Watson, P. L. Are wastewater systems adapting to climate change? J. Am. Water Resour. Assoc. 55, 869-880 (2019).

38. Agrawal, A. \& Carmen Lemos, M. Adaptive development. Nat. Clim. Chang. 5, 185 (2015).

39. Smith, K. R. et al. Human health: impacts, adaptation, and co-benefits. In Climate Change 2014: Impacts, Adaptation, and Vulnerability. Part A: Global and Sectoral Aspects. Contribution of Working Group II to the Fifth Assessment Report of the Intergovernmental Panel on Climate Change (eds. Field, C. et al.). 709-754 (Cambridge University Press, Cambridge, 2014).

40. Anderson, G. B., Barnes, E. A., Bell, M. L. \& Dominici, F. The future of climate epidemiology: opportunities for advancing health research in the context of climate change. Am. J. Epidemiol. 188, 866-872 (2019).

41. Chen, W. H. \& Azman, A. S. Mitigating cholera in the aftermath of cyclone idai. Am. J. Trop. Med. Hyg. https://doi.org/10.4269/ajtmh.19-0285 (2019).

42. WHO/UNICEF JMP. Progress on Household Drinking Water, Sanitation and Hygiene 2000-2017 Special Focus on Inequalities (WHO and UNICEF, Geneva, 2019).

43. Bressler, J. M. \& Hennessy, T. W. Results of an Arctic Council survey on water and sanitation services in the Arctic. Int. J. Circumpolar Health 77, 1421368 (2018).

44. Pauw, W. P. et al. NDC explorer. https://doi.org/10.23661/ndc_explorer_2017_2.0 (2016).

45. Dickin, S. \& Dzebo, A. Missing in climate action: concrete health activities in nationally determined contributions. Lancet Planet. Heal. 2, e144 (2018).

46. Brandi, C., Dzebo, A., Janetschek, H., Lambert, C. \& Savvidou, G. NDC-SDG connections. https://doi.org/10.23661/ndc-sdg_2017_1.0, (2017).

47. Zouboulis, A. \& Tolkou, A. Effect of climate change in wastewater treatment plants: reviewing the problems and solutions. in Managing Water Resources under Climate Uncertainty (eds Shrestha, S., Anal, A. K., Salam, P. A. \& van der Valk, M.), 197-220 (Springer International Publishing, 2015).

48. Hutton, G. \& Varughese, M. The Costs of Meeting the 2030 Sustainable Development Goal Targets on Drinking Water, Sanitation, and Hygiene (World Bank, Washington, 2016).

49. OECD DAC. Climate-related development finance: a bilateral provider perspective. https://public.tableau.com/views/Climate-relateddevelopmentfinance/CRDFDonor?:embed $=\mathrm{y} \&$ :display_count $=$ no\&\%3AshowVizHome $=$ no\%20\#3. Accessed 11 May 2019.

50. UN-Water. Financing Universal Water, Sanitation and Hygiene Under the Sustainable Development Goals: UN-Water Global Analysis and Assessment of Sanitation and Drinking-Water (UN Water, Geneva, 2017).

51. Weikmans, R., Timmons Roberts, J., Baum, J., Bustos, M. C. \& Durand, A. Assessing the credibility of how climate adaptation aid projects are categorised. Dev. Pract. 27, 458-471 (2017).

52. Hutton, G. \& Chase, C. The knowledge base for achieving the sustainable development goal targets on water supply, sanitation and hygiene. Int. J. Environ. Res. Public Health 13, 536 (2016).

53. Parker \& Co., LLC. Liberia WASH Sector Pooled Fund Feasibility Study (Parker \& Co., 2019).

54. Atteridge, A. \& Canales, N. Climate Finance in the Pacific: An Overview of Flows to the Region's Small Island Developing States (SEl, Stockholm, 2017). 
55. Andersson, K. et al. Sanitation, Wastewater Management and Sustainability —From Waste Disposal To Resource Recovery (SEI and UNEP, Stockholm and Nairobi, 2016).

56. MacFadden, D. R., McGough, S. F., Fisman, D., Santillana, M. \& Brownstein, J. S. Antibiotic resistance increases with local temperature. Nat. Clim. Chang 8, 510-514 (2018)

57. Xue, X., Hawkins, T., Schoen, M., Garland, J. \& Ashbolt, N. Comparing the life cycle energy consumption, global warming and eutrophication potentials of several water and waste service options. Water 8, 154 (2016).

58. GIZ and IWA. Introduction to ECAM 2.2: assessing energy performance and carbon emissions within the water sector. https://wacclim.org/ecam/ (2019).

59. GWP and UNICEF. WASH Climate Resilient Development (GWP and UNICEF, Stockholm and New York, 2014).

60. WHO. Operational Framework for Building Climate Resilient Health Systems (WHO, Geneva, 2015).

\section{ACKNOWLEDGEMENTS}

This work was supported by the Swedish International Development Cooperation Agency, through the Stockholm Environment Institute's Sustainable Sanitation Initiative. SIWI authors' contributions were supported by the Swedish International Development Agency through the UNDP-SIWI Water Governance Facility (WGF). We are grateful to Adis Dzebo and Georgia Savvidou for assistance with the NDC-SDG Connections data.

\section{AUTHOR CONTRIBUTIONS}

S.K.D., K.A., A.J. and R.G. conceived the paper. M.B. collected and analysed the data. S K.D. wrote the first draft of the paper with development from R.G. and A.J. on adaptation and from K.A. and M.B. on mitigation. All authors commented on subsequent drafts and revisions.

\section{COMPETING INTERESTS}

The authors declare no competing interests.

\section{ADDITIONAL INFORMATION}

Correspondence and requests for materials should be addressed to S.D.

Reprints and permission information is available at http://www.nature.com/ reprints

Publisher's note Springer Nature remains neutral with regard to jurisdictional claims in published maps and institutional affiliations.

Open Access This article is licensed under a Creative Commons Attribution 4.0 International License, which permits use, sharing, adaptation, distribution and reproduction in any medium or format, as long as you give appropriate credit to the original author(s) and the source, provide a link to the Creative Commons license, and indicate if changes were made. The images or other third party material in this article are included in the article's Creative Commons license, unless indicated otherwise in a credit line to the material. If material is not included in the article's Creative Commons license and your intended use is not permitted by statutory regulation or exceeds the permitted use, you will need to obtain permission directly from the copyright holder. To view a copy of this license, visit http://creativecommons. org/licenses/by/4.0/.

(c) The Author(s) 2020 